\title{
Pathogenic bacteria profile and antimicrobial susceptibility patterns of ear infection at Ayub Medical Complex Abbottabad, Pakistan
}

\author{
Mahnoor Javed, Madiha Arshad and Muhammad Azam Khan* \\ Department of Microbiology Government Post Graduate College Mandian Abbottabad-Pakistan \\ *Corresponding author's email:microbiologistazam@gmail.com \\ Citation \\ Mahnoor Javed, Madiha Arshad and Muhammad Azam Khan. Pathogenic bacteria profile and antimicrobial \\ susceptibility patterns of ear Infection at Ayub Medical Complex Abbottabad, Pakistan. Pure and Applied \\ Biology. Vol. 9, Issue 1, pp714-719. http://dx.doi.org/10.19045/bspab.2020.90077
}

\begin{tabular}{llll}
\hline \hline Received: 06/09/2019 & Revised: 01/12/2019 & Accepted: 09/12/2019 & Online First: 23/12/2019 \\
\hline \hline
\end{tabular}

\section{Abstract}

The aim of this study was to determine the bacterial etiologic agents of ear infections and their antibiogram among patients of all age groups visited to Ayub Medical complex Abbottad. This cross sectional study was designed at Microbiology laboratory, Ayub Medical complex Abbottabad from February 2019 to July 2019. Patients of any age and both sexes were involved. For the culturing of bacteria and susceptibility testing swabs from infected ears were analyzed. Anitbiogram was perform according to CLSI standards. A total of 100 samples were collected. Of them, $(55 \%)$ were males and $(45 \%)$ female patients. Overall, $(72 \%)$ samples were positive in which $(76 \%)$ were male. Highest number of isolates $20(76 \%)$ were identified in patients from 1-10 years of age. The predominant bacteria were P. aeruginosa $32(44.4 \%)$, S. aureus $15(20.83 \%)$ and K.pneumoniae 8 (11.11\%). Gram positive bacterial isolates exhibited high level of resistance to Tetracycline, Penicillin, Oxacillin and Vancomycin respectively while Cotrimoxazole, Clindamycin, Ciprofloxacin, Linezolid, Erythromycin and chloramphenicol were effective drugs. Gram negative bacteria were resistant to Amoxicillin and Ampicillin. However, Amikacin, Pipra-tazobactum, Ceftriaxone, Gentamicin, Ciprofloxacin, Chloramphenicol and Cotrimoxazole were effective against most of these isolates. Bacteriological Ear infection is reasonably common in Abbottabad and the most pathogenic bacteria associated with infection are P.seudomonas S.aureus and K.pneumoniae which are resistant to Beta Lactam antibiotics. Therefore, for the cure of ear infection antibiogram is vital.

Keywords: Ear infection; Pathogenic bacteria; P.seudomonas; S.aureus; Antibiogram

\section{Introduction}

The infection of middle ear or tympanic tissue is termed as otitis media (OM) which is characterized by ear irritation or ear discharge (otorrhae) [1]. Ear infection may be acute or chronic purulent type [2]. Approximately 330 million peoples are suffered from ear infection throughout the world in which about $60 \%$ lost hearing capability [3]. Both in developing and developed countries its high prevalence has created problems for public health [4]. Mainly ear infection is common in young children and infants but also affect adults as well [5]. The infection may initiate in babyhood due to unnoticed treatment [4]. The microorganisms may spread to the middle ear through a long-lasting 
perforation. As compared to adults children are at risk of ear infection due to non-developed Eustachian tube in children allows easier entrée of organism over the nasopharynx. Besides, the ratio of infection is higher in men than in women [6]. The frequency of otitis media fluctuates from region to region. In the advanced countries, it is decreasing due to wakefulness; but in emerging world, it is on the upsurge [7]. In developed countries untreated ear problems leads to middle ear infection often with impairment and more difficulties comprising persistent acute otitis media, persistence of middle ear inflammation which requires the insertion of drainage tube and often results in loss of hearing and chronic middle ear infection [6]. The causative agents, incidence and antibiogram of ear diseases is varied with environmental area and meteorological conditions surroundings [2]. A number of studies, documented that Staphylococcus aureus, Escherichia coli, Pseudomonas aeruginosa, Klebsiella pneumoniae and Proteus mirabilis are the topmost organisms isolated from circumstances of ear illnesses [8]. Wide-spectrum antibiotics are used in huge amount and in inappropriate way that has triggered difficulties to treat the bacterial isolates which became worldwide wellbeing risk [2]. In ear infection high numbers of antibiotic are used to treat bacteria results in the initiation of secondary infections of brain [9]. In addition, ear infections can cause the formation of middle ear tumors, auditory inflammation and auditory problems $[9,10]$. The prevalence, antibiotic resistance and causative agents of ear infection from region to region varies due to differences in antibacterial recommending practices and incidence of resistant bacterial strains. Thus up to date information on microbial resistance need to be available at national and local level to guide the rational use of existing antimicrobials. Therefore, this study was carried out to determine bacterial etiologic agents of ear infections and their antimicrobial susceptibility profiles among patients who referred to Ayub Medical Complex Abbottabad.

\section{Materials and methods}

Area of study

This cross sectional study was designed at Microbiology section, Pathology Department, Ayub Medical complex Abbottabad from February 2019 to July 2019.

\section{Sample collection}

A total of 100 patients of suspecting otitis media presenting with ear discharge were involved in the study. Patients of any age and both sexes were involved. Auditory swabs wetted with sterilized normal saline were used to assemble samples and transported directly to lab for culture. Swabs were reserved prior start medical treatment.

\section{Sample processing for isolation of pathogenic bacteria}

Discharge swabs were cultivated on blood agar, MacConkey agar and Chocolate agar. Swabs were processed using standard bacteriological methods to isolate bacteria and identify growing organisms according to standard bacteriological procedures. [11].

\section{Antibiotic susceptibility testing}

After 72 hours of incubation, all cases of bacterial growth were tested for antibiotic susceptibility using a modified Kirby Bauer disk diffusion method and analyzed using standard guidelines. [12].

\section{Antibiotic used in the study}

The antibiotic tested in the study against the isolated bacteria are Clindamycin, Oxacillin, Tetracycline, Chloramphenicol, Erythromycin, Penicillin, Linezolid, Vancomycin Amoxicillin, Ampicillin, Ciprofloxacin, Ceftriaxone, Cotrimoxazole Gentamycin, Amikacin, Pipra-tazobactum, Ceftazidin, Levofloxacin,

\section{Results}

Overall 100 specimens from patients of otitis media were evaluated both from men $55(55 \%)$ and female $45(45 \%)$. They were (range from 1 to above 30) years. Total, $72(72 \%)$ of otitis media had 
bacteriological isolates. The ratio of infection was $42(76 \%)$ in men and 30 (66 $\%)$ in female. The incidence isolates were identified in $20(76 \%) 25(73.3 \%)$, and $27(67 \%)$ patients from 1 to 10,21 to 30 , and 11 to 20 years of age, respectively (Table $1)$.

Of 72 isolates, Gram negative bacteria were $52(72.2 \%)$ in contrast to Gram positive 20 $(27.7 \%)$. So a total of nine bacteria were isolated. The predominant bacteria were $P$. aeruginosa 32 (44.4\%) S. aureus 15 (20.83 $\%)$ and K.pneumoniae 8 (11.11\%), E.coli (6.94\%) S.pneumoniae (4.16\%) Acinetobacter $(4.16 \%)$ and Proteus $(2.77 \%)$ as tabulated in (Table 2).

Mostly, gram positive isolates exhibited $(9.9-90.0 \%) \quad$ resistance against the antibiotics used. Out Of $15 \mathrm{~S}$. aureus isolates, (90.0, 39.0, 32.0 and $26 \%)$ were resistant to Penicillin, Tetracycline Oxacillin and Vancomycin correspondingly. But resistance was minimum against Cotrimoxazole, Clindamycin, Ciprofloxacin, Linezolid, Erythromycin and Chloramphenicol by Gram positive bacteria (Table 3 ).

Gram negative bacteria were (6.0-91.0\%) resistance to the antimicrobials practiced. $P$. aeruginosa, Proteus spp., E. coli and $K$. pneumoniae Acinetobacter were resistant to Amoxicillin/Clavulanic acid (60-80\%) and Ampicillin (85-100 \%). On the other side, Amikacin, Pipra-tazobactum, Ceftriaxone, Gentamycin, Ciprofloxacin, Chloramphenicol and Cotrimoxazole were effective against Gram negative bacterial isolates (Table 4).

Table1. Infection of ear in relation to age and gender of patients visited at Ayub Medical Complex Abbottabad

\begin{tabular}{|c|c|c|}
\hline Variables & Ear infection N (\%) & Total N (\%) \\
\hline Male & $42(76.36)$ & $55(55)$ \\
\hline Female & $30(66.66)$ & $45(44)$ \\
\hline Age (years) & & $26(26)$ \\
\hline $1-10$ & $20(76.92)$ & $40(40)$ \\
\hline $11-20$ & $27(67.5)$ & $34(34)$ \\
\hline $21-30$ & $25(73.53)$ & $100(100)$ \\
\hline Total & 72 & \\
\hline
\end{tabular}

Table 2. Frequency of bacterial isolates $(n=72)$ of ear infection at Ayub Medical Complex Abbottabad

\begin{tabular}{|c|c|c|c|}
\hline \multicolumn{4}{|c|}{ Bacteriological profile } \\
\hline Bacterial isolates & Total N=72 & Percentage \\
\hline \multirow{3}{*}{ Gram positive bacteria } & S.pneumonia & 3 & 4.16 \\
\cline { 2 - 4 } & S.aureus & 15 & 20.83 \\
\cline { 2 - 4 } & Enterococcus & 2 & 2.77 \\
\hline \multirow{5}{*}{ Gram negative bacteria } & P. aeruginosa & 32 & 44.44 \\
\cline { 2 - 4 } & E.coli & 5 & 6.94 \\
\cline { 2 - 4 } & K.pneumoniae & 8 & 11.11 \\
\cline { 2 - 4 } & Acinetobacter & 3 & 4.16 \\
\cline { 2 - 4 } & Proteus & 2 & 2.77 \\
\cline { 2 - 4 } & Citrobacter & 2 & 2.77 \\
\hline
\end{tabular}


Table 3. Antibacterial resistance patterns of Gram positive bacterial isolates $(n=20)$ from pus swab taken from ears infection of patients, at Ayub Medical Complex Abbottabad

\begin{tabular}{|c|c|c|c|c|c|c|c|c|c|c|c|}
\hline $\begin{array}{c}\text { Isolated } \\
\text { bacteria }\end{array}$ & \multicolumn{10}{|c|}{ Resistant pattern of antibacterial agents (R \%) } \\
\hline & $*$ T & DA & OXA & TE & SXT & CAF & CIP & ERY & PEN & LZD & VA \\
\hline S. aureus & 15 & 10 & 32 & 39 & 20 & 5.4 & 0.0 & 20 & 90 & 0.0 & 26 \\
\hline S. pneumonia & 3 & ND & ND & 9.9 & 0.0 & 2 & 0.0 & 0.0 & 20 & 0 & 0 \\
\hline Enterococcus & 2 & 290 & ND & ND & ND & 0 & 0 & ND & ND & 50 & ND \\
\hline Total & 20 & & & & & & & & & & \\
\hline
\end{tabular}

Key: \#T total number of bacteria experienced against each antibacterial agent, $R \%$ percent of bacteria resistance to antimicrobial agent, $N D$ not done, $D A$ Clindamycin, $O X A$ Oxacillin, $T E$ Tetracycline, $S X T$ Cotrimoxazol, $C A F$ chloramphenicol, CIP ciprofloxacin, ERY Erythromycin, PEN penicillin, LZD Linezolid, VA Vancomycin

Table 4. Antibacterial resistance patterns of Gram negative bacteria $(n=52)$ isolated from patients of ears infection, at Ayub Medical Complex Abbottabad

\begin{tabular}{|c|c|c|c|c|c|c|c|c|c|c|c|}
\hline $\begin{array}{c}\text { Isolated } \\
\text { bacteria }\end{array}$ & \multicolumn{10}{|c|}{ Resistant pattern of antimicrobial agents(R \%) } \\
\hline & $*$ T & AMC & AMP & CIP & CRO & SXT & GEN & AK & TZP & CAZ & LEV \\
\hline P. aeruginosa & 52 & 80 & 100 & 6 & 18 & 20.1 & 10 & 6 & 5 & 15 & 9 \\
\hline K. pneumonia & 8 & 70 & 100 & 12 & 18 & 13 & 10 & 3.0 & ND & 25 & 0.0 \\
\hline E.coli & 5 & 69 & 89.1 & 10 & 30 & 0.0 & 35 & 0.0 & 0.0 & 20 & 0.0 \\
\hline Acinetobacter & 3 & 88 & 85 & ND & ND & ND & ND & 2.5 & ND & ND & ND \\
\hline Proteus $S P P$ & 2 & 60 & 90 & 10 & 25 & 20 & 26 & 0.0 & 0.0 & 26 & ND \\
\hline Citrobacter & 2 & ND & ND & ND & ND & ND & ND & ND & 90 & ND & ND \\
\hline Total & 72 & & & & & & & & & & \\
\hline
\end{tabular}

Key: \#T total bacteria tested against each antibiotic, $R \%$ percent of isolates resistant to antimicrobial agent, AMC amoxicillin, AMP Ampicillin, CIP Ciprofloxacin, CRO Ceftriaxone, SXT Cotrimoxazole GEN Gentamycin, AK Amikacin, TZP Pipra-tazobactum, , CAZ Ceftazidin, Lev Levofloxacin , ND not done

\section{Discussion}

Patients use antibiotics and make appointment with clinicians for the treatment of disturbing ear infection [20]. For diagnostic purpose, ear discharge sample is one of the most commonly desired sample from medical sites. Correspondingly, our study, $72.0 \%$ specimens of infected ear displayed bacterial growth. Similarly, with an insignificant dissimilarity few former authors in Ethiopia reported $89.5 \%$ [8], $89.4 \%$ and [6]. This designates that otitis media is problematic to public health and humanity. In the current research ear infection was suggestively greater in man with comparison to females this is similar to consequences of [14]. But dissimilarity was reported by the study of females had higher ratio of ear infection than males [13]. In this study, the otitis media was greater $(76.92 \%)$ in people from 1 to 10 years of age which come to an agreement with data from $[10,17]$. Greater occurrence of otitis amongst undeveloped age individuals might be due to the small, wide and orthodox nature of the Ear channel, absence of sanitization, weak immunity, frequent contact to upper respiratory tract infections and undernourishment [10]. In our study, the most significant bacteria were gram-negative $52(72.2 \%)$ of ear discharge associated to gram-positive bacteria which is parallel to previously studies in Dessie [9] and Ethiopia [14].

In the current research, $P$. aeruginosa $(44.44 \%)$ tailed by $S$. aureus $(20.83 \%)$ and Proteus spp. (2.77 \%). This fashion is analogous to studies of other scientists [14, 16, 21]. However the finding of Denboba [22] showed contrast to our results. The reason of differences in bacterial profile of ear infection is due to deviation in type of weather and natural features, further nationwide study is important. The most dominant isolate in our study was of $P$. 
aeruginosa, could be associated to its potential of survival in antagonism with other creatures and fight to antibiotics. Besides, $P$. aeruginosa has virulence factors such is Pilli which helps in the attachment of the pathogen to the epithelial layer of inflamed middle ear. When involved, the pathogen yields proteins degrading enzymes to evade the usual resistance procedure of organ vital to combat infections [6].

The occurrence of $K$. pneumoniae and $E$. coli in this research was 11.11 and $6.94 \%$, respectively. Similarly, 11.1 and $3.7 \%$ of $K$. pneumoniae and $E$. coli was, correspondingly described in Ethiopia [2]. However, a study in India [18] described 8 and $4 \%$ of $K$. pneumoniae and E. coli, separately although the percentage differs. Separation of fecal bacteria like $K$. pneumonia and $E$. coli might designate that personages were at danger of problems due to underprivileged cleanliness environments. In this study $P$. aeruginosa exhibited extraordinary level of resistance (80-100 \%) to ampicillin and amoxicillin. This was parallel with results in ethopia [14] where 90-89 the levels of resistance to Ampicillin and Amoxicillin were observed separately. However, Pseudomonas aeruginosa is highly susceptible to ciprofloxacin, chloramphenicol, gentamicin and amikacin. This is similar to the Ethiopian results. [2].

In the current study, $90 \%$ and $36 \% \mathrm{~S}$. aureus growth were resilient to Penicilin and Oxacilin, correspondingly. These findings were similar to previous studies [14, 19]. This recommends the dissemination of all $S$. aureus not sensitive to every $\beta$-lactem medicines and Methicilln to public which offers a vigilant for extra huge balance research on the occurrence and vulnerability patterns of public assimilated Methicilln resistant S. aureus middle ear disease in Abbottabad. Additional, S. aureus showed minimum resistance to Ciproflaxacin, Chloramphenical, Clindamcin, Erythromycen and Cotrimaxazole. These were same to the outcomes of other studies $[6,9]$ for Ciproflxacin and Erythromcin. In the existing study, Proteus spp. displayed extraordinary resistance $(80 \%)$ to ampecillin and adequate resistance (60\%) to amoxcilin/clavulenic acid. Though, Proteus spp. shown low levels of resistance to ciproflxacin, gentamycin and ceftriaxone which were similar to former results $[2,6$, 8, 9]. The existence of high levels of resistance to beta lactam antibiotics and the bacteria linked with internal ear dysfunctions in our research may be due to absence of study on antibacterial resistance amongst doctors and indulges, inaccessibility of inadequate antibiogram minutes, exploitation of medicines, and self-use and carelessness on patient part.

\section{Conclusion}

The study indicates that ear infection is most common in Abbottabad. The major bacteria involved in infection are $P$. aeruginosa, S. aureus and K.pneumoniae. Most of the isolates were resistant to amoxicillin/clavulanic acid and ampicillin and oxacillin. However, Amikacin, cotrimoxazole, ciprofloxacin, chloramphenicol and gentamicin were effective against most of the bacterial isolates. Therefore, for the treatment of otitis media in the study area antibiogram is essential.

\section{Authors' contributions}

Prepared and supervised the research project: MA Khan, Performed the experiment and helped in proofreading of the manuscript: $\mathrm{M}$ Javeed \& M Arshad.

\section{Acknowledgement}

The study was done at Ayub medical complex Abbottabad. Thank Dr UZMA for her support to complete this research.

\section{References}

1. Worku M \& Bekele M (2014). Bacterial isolate and antibacterial resistance pattern of ear infection among patients attending at Hawassa university referral Hospital, Hawassa, Ethiopia. Indian J of Otol 20(4): 155.

2. Wasihun AG \& Zemene $Y$ (2015). Bacterial profile and antimicrobial susceptibility patterns of otitis media in Ayder Teaching and Referral Hospital, 
Mekelle University, Northern Ethiopia. Springer Plus 4(1): 701.

3. Woodfield G \& Dugdale A (2008). Evidence behind the WHO guidelines: hospital care for children: what is the most effective antibiotic regime for chronic suppurative otitis media in children. $\mathbf{J}$ of Tropical Pediatrics 54(3): 151-156.

4. Afolabi OA, Salaudeen AG, Ologe FE, Nwabuisi C \& Nwawolo CC (2012). Pattern of bacterial isolates in the middle ear discharge of patients with chronic suppurative otitis media in a tertiary hospital in North central Nigeria. African Health Sci 12(3): 362-367.

5. Abdullah FE, Khatri PK, Alzadjali NA, Ali AD \& Bhagia G (2011). Ear infections in Karachi: The frequency and antibiotic resistance of bacterial isolates.

6. Seid A, Deribe F, Ali K \& Kibru G (2013). Bacterial otitis media in all age group of patients seen at Dessie referral hospital, North East Ethiopia. Egyptian $J$ of Ear, Nose, Throat and Allied Sci 14(2): 73-78.

7. Adoga AS, Maan EN, Malu D, Badung BP, Obiesie IV \& Nwaorgu OGB (2010). Swab and aspiration specimen collection methods and antibiogram in chronic suppurative otitis media at Jos University Teaching Hospital: Which is superior. Annals of African Med 9(4).

8. Muluye D, Wondimeneh Y, Ferede G, Moges F \& Nega T (2013). Bacterial isolates and drug susceptibility patterns of ear discharge from patients with ear infection at Gondar University Hospital, Northwest Ethiopia. BMC Ear, Nose and Throat Disorders 13(1): 10.

9. Abera B \& Kibret M (2011). Bacteriology and antimicrobial susceptibility of otitis media at dessie regional health research laboratory, Ethiopia. Ethiopian J of Health Devel 25(2): 161-167.

10. Ahmad S (2013). Antibiotics in chronic suppurative otitis media: A bacteriologic study. Egyptian Journal of Ear, Nose, Throat and Allied Sci 14(3): 191-194.

11. Forbes BA, Sham DF, Weissfeld AS, Forbes BA. (2007) Bailey \& Scott's Diagnostic Microbiology (12th Ed.), pp 778-781.

12. Wayne PA (2011). Clinical and laboratory standards institute. Performance standards for antimicrobial susceptibility testing.
13. Hassan O \& Adeyemi RE (2007). A study of bacterial isolates in cases of otitis media in patients attending oauthc, IleIfe. African $J$ of Clinical and Exper Microbiol 8(3): 130-136.

14. Hailu D, Mekonnen D, Derbie A, Mulu W \& Abera B (2016). Pathogenic bacteria profile and antimicrobial susceptibility patterns of ear infection at Bahir Dar Regional Health Research Laboratory Center, Ethiopia. SpringerPlus 5(1): 466.

15. Iseh KR \& Adegbite T (2004). Pattern and bacteriology of acute suppurative otitis media in Sokoto, Nigeria. Ann Afri Med 3(4): 164-166.

16. Fatima G, Shoaib M, Raza MZ \& Bilal S (2013). Antimicrobial susceptibility pattern of bacterial and fungal isolates from patients with chronic suppurative otitis media in perspective of emerging resistance. Pak J Otolaryngo 29: 49-53.

17. Basnet R, Sharma S, Rana JC \& Shah PK (2017). Bacteriological study of otitis media and its antibiotic susceptibility pattern. $J$ of Nepal Health Res Council 15(2): 124-129.

18. Prakash M, Lakshmi K, Anuradha S \& Swathi GN (2013). Bacteriological profile and their antibiotic susceptibility pattern of cases of chronic suppurative otitis media. Asian J Pharm Clin Res 6(3): 210212.

19. Hwang JH, Tsai HY \& Liu TC (2002). Community-acquired methicillin-resistant Staphylococcus aureus infections in discharging ears. Acta Otolaryngologica 122(8): 827-830.

20. Grevers $G$ (2010). Challenges in reducing the burden of otitis media disease: an ENT perspective on improving management and prospects for prevention. Inter $J$ of Pediatric Otorhinolaryngol 74(6): 572577.

21. Aslam MA, Ahmed Z \& Azim R (2004). Microbiology and drug sensitivity patterns of chronic suppurative otitis media. $J$ of the College of Physicians and Surgeons-Pakistan: JCPSP 14(8): 459-461.

22. Argaw-Denboba A, Abejew AA \& Mekonnen AG (2016). Antibiotic-resistant bacteria are major threats of otitis media in Wollo Area, Northeastern Ethiopia: a tenyear retrospective analysis. Inter $J$ of Microbiol. 